\title{
ERRATUM
}

Yukihiro Morimoto $\cdot$ Yosihiro Natuhara

Atsuo Morimura $\cdot$ Masahiro Horikawa

\section{The pelican scenario for nature restoration of Aral Sea wetland ecosystems}

Published online: 25 June 2005

(C) International Consortium of Landscape and Ecological Engineering and Springer-Verlag Tokyo 2005

\section{Landscape Ecol Eng (2005) 1:85-92}

Throughout the article, "superimposed" should read "supervised." It appears:

p.86 right column, line 17

p.88 left column, lines 5 and 7; legends for Figs. 2 and 3

p.89 right column, line 13 from the bottom

The online version of the original article can be found at http:// dx.doi.org/10.1007/s11355-005-0008-y

Y. Morimoto $(\square)$

Graduate School of Global Environmental Studies,

Kyoto University, Kitashirakawa Oiwake, Sakyo-ku,

Kyoto 606-8502, Japan

E-mail: ymo@kais.kyoto-u.ac.jp

Y. Natuhara $\cdot$ M. Horikawa

Graduate School of Agriculture and Biological Sciences,

Osaka Pref. University, Sakai, Japan

A. Morimura

Faculty of Human Environments,

University of Human Environments, Okazaki, Japan 